\title{
GATE (Gaia Transiting Exoplanets): Detecting Transiting Exoplanets with Gaia
}

\author{
Shay Zucker ${ }^{1}$, Laurent Eyer ${ }^{2}$, Simon Hodgkin ${ }^{3}$ and Gisella \\ Clementini $^{4}$
}

${ }^{1}$ Dept. of Geosciences, Faculty of Exact Sciences, Tel Aviv University, Tel Aviv, Israel ${ }^{2}$ Dept. of Astronomy, University of Geneva, Geneva, Switzerland ${ }^{3}$ Institute of Astronomy, Cambridge University, Cambridge, UK ${ }^{4}$ INAF - Osservatorio Astronomico di Bologna, Bologna, Italy

Gaia will have a revolutionary impact on most fields of astronomy. However, its scanning law is too sparse for traditional transit detection approaches (de Bruijne 2012). Practically, only stars brighter than 16th magnitude are relevant for follow-up of transiting exoplanets. For those stars, Gaia's precision is of the order of 1 mmag (Eyer et al. 2015). On average, Gaia will have sampled each target 70 times, but certain stars may be observed as many as 200 times (Voss et al. 2013). Hipparcos scanning law was similar, but its precision much worse. Nevertheless the transit of HD209458 could be seen, aposteriori, in Hipparcos' data (Söderhelm 1999). This inspired our GATE initiative.

For each star, GATE will use Gaia data and Bayesian inference to produce probability distributions of potential transits. These distributions will be used to infer Instantaneous Transit Probability (ITP) - the probability for a hypothetical transit to occur in each future time (Dzigan \& Zucker 2011 and 2013). ITP will be calculated by Coordination Unit 7 (CU7) of Gaia DPAC (Data processing and Analysis Consortium). The times of highest ITP are the best times to observe a star, in order to determine if transits do occur. Best times will be disseminated to the community using the facilities of Gaia Science Alerts (GSA). GSA operates a website, a Twitter account, and other channels, to alert the astronomical community about variable sources to follow (Hodgkin et al. 2013).

GATE will use the DPAC/CU7 network of telescopes for Supplementary Observations. Participation of the global observer community, using the GSA alerts will improve significantly the detection yield of GATE. All observatories with the ability to observe transits of extrasolar giant planets are welcome to contribute their observing power.

GATE is essential for full exploitation of Gaia's potential to detect transiting exoplanets. Using GATE, we estimate that Gaia will be able to detect a few thousand transiting exoplanets. It is important to start observations as soon as alerts become possible, since the information contained in Gaia data will degrade over time. The DFU approach can be utilized to other low-cadence photometric surveys, such as Pan-STARRS, ZTF, NGTS, etc.

\section{References}

de Bruijne, J. 2012, Ap\&SS, 341, 68

Dzigan, Y. \& Zucker, S. 2011, MNRAS, 415, 2513

Dzigan, Y. \& Zucker, S. 2013, MNRAS, 428, 3641

Eyer, L., et al. 2015, in Living Together Planets, Host Stars and Binaries, eds. S.M. Rucinski, G. Torres \& M. Zejda, ASP Conf. Ser V. 496 (San Francisco: ASP), p. 121

Hodgkin, S., et al. 2013, Phil. Trans. R. Soc. A, 371, 20239

Söderhelm, S. 1999, Inf. Bull. Var. Stars, 4816, 1

Voss, H., et al. 2013, in Highlights of Spanish Astrophysics VII, eds. J.C. Guirado, L.M. Lara, V. Quilis \& J. Gorgas, p. 738 